\title{
Características psicométricas da Relationship Assessment Scale
}

\author{
Vicente Cassepp-Borges' 1 - Universidade Federal da Grande Durados, Dourados, Brasil \\ Luiz Pasquali - Universidade de Brasilia, Brasilia, Brasil
}

\begin{abstract}
Resumo
Em nível internacional, a Relationship Assessment Scale (RelAS) é um dos principais testes para avaliar satisfação no relacionamento. É um teste unifatorial com sete itens. Este estudo teve por objetivo apresentar as propriedades psicométricas da escala no Brasil. O teste foi aplicado em uma amostra de 1.549 participantes de 12 estados brasileiros e do Distrito Federal. A maioria dos dados foi coletada em universidades. Os dados foram analisados de acordo com a Teoria Clássica dos Testes e com a Teoria de Resposta ao Item. A estrutura unifatorial e a boa precisão $(\alpha=0,85)$ foram replicadas, e a escala apresentou itens discriminativos (parâmetro $a$ ). A maioria das dificuldades dos itens (parâmetro $b$ ) foram fáceis e médias, o que levou a escala a ser informativa para sujeitos abaixo do percentil 80. A RelAS é um instrumento simples, curto e útil para avaliar a satisfação no relacionamento também no Brasil.

Palavras-chave: Estados emocionais, Relações conjugais, Teoria de Resposta ao Item, Satisfação, Comportamento Psicossexual.
\end{abstract}

Relationship Assessment Scale's psychometric characteristics

\begin{abstract}
The Relationship Assessment Scale (RelAS) is one of the main tests to assess the relationship's satisfaction used worlwide. This is a single-factor test with seven items. This present study aimed to show the psychometric properties of the scale in Brazil. The test was applied on 1549 participants from 12 Brazilian states and the Federal District. Most of the data were collected in universities. Data were analyzed according to the Classical Theory of Tests and the Item Response Theory. Single-factor structure and good reliability $(\alpha=0,85)$ were replied and the scale had discriminative items (parameter $a$ ). Most of the difficulties of the items (parameter $b$ ) were easy and medium, which made the scale informative for subjects below 80 percentile. The RelAS is a simple, short, and useful instrument to assess the satisfaction in the relationship also in Brazil.

Keywords: Emotional states, Marital relations, Item Response Theory, Satisfaction, Psychosexual behavior.
\end{abstract}

A satisfação no relacionamento é um dos construtos que mais vem sendo trabalhados na área de avaliação dos relacionamentos (Hendrick, 1988). Mais especificamente, a investigação de relações entre amor e satisfação no relacionamento tem sido um tema de pesquisa frequente no exterior (Masuda, 2003). A satisfação no relacionamento é uma avaliação subjetiva bastante próxima ao bem-estar (Gable \& Poore, 2008). Refere-se a uma avaliação cognitiva positiva do relacionamento. Além de ser avaliada no nível individual, trata-se de comparação de outras relações com a sua própria. Este conceito engloba relacionamentos de casal, noivos, namorados, "ficantes", ou mesmo relacionamentos sem compromisso (Wachelke, De Andrade, Cruz, Faggiani \& Natividade, 2004). A satisfação conjugal pode ser definida como uma atitude frente tanto à interação conjugal quanto às características do(a) cônjuge (Dela Coleta, 1989).

A satisfação no relacionamento tem sido um tema de pesquisa relativamente frequente no Brasil. Em uma amostra de 106 participantes, foi emcontrada uma

\footnotetext{
${ }^{1}$ Endereço para correspondência:

Universidade Federal da Grande Dourados - Unidade II Faculdade de Ciências Humanas, gabinete 16 - Rodovia Dourados Itahum, Km 12, Caixa Postal - 533 - 79.804-970 - Dourados-MS Brasil
}

correlação pequena e positiva entre o construto e a satisfação com a vida (Scorsolini-Comin \& Santos, 2010a). Em uma pesquisa com 38 casais paulistas, verificou-se que a proximidade, boas estratégias para resolver problemas, coesão, capacidade de comunicação, satisfação com a condição econômica e a prática religiosa são variáveis preditoras da satisfação conjugal (Norgren, Souza, Kaslow, Hammerschmidt \& Sharlin (2004). Em outro estudo, realizado com 362 participantes na capital gaúcha, verificou-se que as três dimensões do amor (intimidade, paixão e compromisso) são fortes preditoras da satisfação no relacionamento $\quad\left(R^{2}=0,54\right) \quad$ (Cassepp-Borges \& Teodoro, 2009).

Mesmo que teorias estejam sendo construídas para compreender o amor, estudar a satisfação nos relacionamentos é algo de importância semelhante. Mensurar $O$ amor nos mostra o tamanho do sentimento, mas a satisfação no relacionamento mostra se isso é bom ou se é ruim. Num casamento, por exemplo, é interessante que se mantenham níveis elevados de amor e satisfação conjugal, pois tem-se ambos como elementos de uma relação bem-sucedida. No caso de um amor platônico, por outro lado, níveis alterados de amor podem trazer insatisfação com o relacionamento, pois um amor muito elevado por alguém que não corresponda pode levar a um alto nível de sofrimento psíquico. Esses exemplos ainda mostram 
que é possível avaliar a satisfação no relacionamento inclusive em relações que não a de casal, como as familiares e de amizade. Para quaisquer pessoas, o amor parece ser o principal preditor da satisfação no relacionamento (Cassepp-Borges \& Teodoro, 2009; Lemieux \& Hale, 2000).

Existem diversos instrumentos para a mensuração deste construto. A Escala de Ajuste Diádico (Dyadic Adjustment Scale - DAS) foi desenvolvida por Spanier (1976) e possui 32 itens que devem ser respondidos por casais que moram juntos. A análise fatorial dessa escala distinguiu claramente os fatores Consenso da díade ( $\alpha=0,90,13$ itens), Satisfação da díade $(\alpha=0,94$, 10 itens), Coesão da díade ( $\alpha=0,86,5$ itens) e Expressão da afetividade $(\alpha=0,73,4$ itens $)$ e demonstrou consistência interna geral $(\alpha=0,92,32$ itens). Cabe salientar que a $D A S$ mede ajustamento da díade, e a satisfação no relacionamento é apenas um dos elementos deste ajustamento. Essa escala vem sendo bastante utilizada, e uma busca em bases de dados encontrou pesquisas recentes em amostras dos cinco continentes, em países como China (Shek \& Cheung, 2008), África do Sul (Lesch \& Engelbrecht, 2008), França (Antoine, Christophe \& Nandrino, 2008), Alemanha (Dinkel \& Balck, 2006), Austrália (Hundertmark, Esterman, Ben-Tovim, Austin, \& Dougherty, 2007) e Estados Unidos (Funk \& Rogge, 2007). Embora não sejam relatados dados psicométricos do instrumento, a $D A S$ foi utilizada no Brasil por Perlin (Perlin, 2001, 2006; Perlin \& Diniz, 2005). Hernandez (2008), em uma amostra portoalegrense, encontrou os mesmos fatores com itens carregando em mais de um fator, o que o autor atribuiu à inter-relação entre as subescalas. Os coeficientes alfas de Cronbach variaram de 0,62 a 0,86 , o que sugere que a escala também precisa ser melhorada em alguns fatores.

Wachelke e colaboradores (2004) desenvolveram a Escala Fatorial de Satisfação em Relacionamento, um instrumento de nove itens (dois inversos) e dois fatores (Satisfação com a Atração Física e Sexualidade - SAFS - e Satisfação com Afinidades de Interesses e Comportamentos - SAIC). A escala foi aplicada em 364 participantes de Porto Alegre e Florianópolis envolvidos(as) em relacionamentos. Apesar dos itens terem apresentado cargas fatoriais adequadas (maiores que 0,30$)$, os valores de alfa $\left(\alpha_{\mathrm{SAFS}}=0,76\right.$ e $\left.\alpha_{\mathrm{SAIC}}=0,61\right)$ indicaram problemas em relação à sua confiabilidade. Em um segundo estudo (Wachelke, De Andrade, Souza \& Cruz, 2007), o instrumento teve um item reformulado por uma questão semântica e foi aplicado em outros 342 universitários. As cargas fatoriais foram maiores que 0,48 , com exceção de um item retirado do instrumento. Os valores alfas também melhoraram
$\left(\alpha_{\mathrm{SAFS}}=0,80\right.$ e $\left.\alpha_{\mathrm{SAIC}}=0,68\right)$, apesar de não se poder afirmar que a escala encontrou total confiabilidade. Porém, em uma aplicação via internet com 545 sujeitos, obteve-se alfa de 0,78 para a SAFS e 0,76 para a SAIC (De Andrade \& Wachelke, 2006).

No Brasil, no entanto, os estudos com instrumentos de medida da satisfação no relacionamento se resumem a essas duas escalas. Ambas tem o problema de possuir diversos itens e uma estrutura fatorial complexa, fugindo ao princípio da parcimônia. Os níveis de precisão, embora aceitáveis, não são os mais indicados para utilização no contexto prático. Diante disso, e considerando os resultados obtidos com a Relationship Assessment Scale (RelAS) no exterior, considerou-se a possibilidade de utilizá-la no Brasil.

A RelAS é uma medida simples da satisfação no relacionamento, com apenas sete itens e estrutura unifatorial. Todos os itens estão em uma escala tipo Likert, variando de 1 a 7, devendo os itens RelAS_4 e RelAS_7 ter os seus escores invertidos $(1=7,2=6, \overline{3}=5$, $4=4,5=3,6=2$ e $7=1$ ) para fazer o somatório com os demais. Apesar de ter poucos itens, a RelAS demonstrou um bom nível de consistência interna $(\alpha=0,86)$ em estadunidenses (Hendrick, 1988). A confiabilidade teste-reteste em uma amostra de 65 universitários, também estadunidenses, com um intervalo de seis a sete semanas, foi de 0,85 (Hendrick, Dicke \& Hendrick, 1998).

Os estudos com a RelAS têm mostrado consistência nas suas propriedades psicométricas. $\mathrm{Na}$ Alemanha, a escala foi estudada em uma amostra de148 participantes, todos(as) casados(as) ou morando com $o$ (a) parceiro(a). A escala mostrou estrutura de um fator e teve cargas superiores a 0,60 , com um elevado nível de confiabilidade $(\alpha=0,89)$ (Dinkel \& Balck, 2005). A estrutura unifatorial também foi encontrada no Reino Unido, com uma amostra de 116 estudantes com idade entre 18 e 21 anos. O nível de confiabilidade foi semelhante aos encontrados na literatura $(\alpha=0,85)$ (Cramer, 2006). Esses estudos exemplificam a estabilidade do fator único e da precisão da RelAS em diferentes contextos.

O principal objetivo deste estudo foi verificar evidências de validade e precisão da RelAS no Brasil, a validade por meio de sua estrutura fatorial, e a precisão pelo alfa de Cronbach e o lambda de Guttman. Não existe nenhum teste para medir a satisfação no relacionamento à disposição do(a) psicólogo(a) brasileiro(a) (CFP, 2011). A presente revisão de literatura não encontrou qualquer utilização da RelAS no Brasil, o que faz com que estudos sobre a escala sejam necessários. Também se teve o objetivo de verificar a discriminação e a dificuldade dos itens da 
escala, de acordo com a Teoria de Resposta ao Item (TRI).

\section{Método}

\section{Participantes}

Este estudo teve a participação de 1.549 pessoas. A média de idade foi de 25,17 anos ( $D P=7,74)$. Os(as) participantes eram oriundos(as) de doze estados brasileiros e do Distrito Federal. As coletas de dados foram realizadas em pelo menos um terço das Unidades da Federação de cada uma das cinco regiões geográficas do Brasil (Instituto Brasileiro de Geografia e Estatística [IBGE], 2011). A Região Centro-Oeste foi a que contou com maior número de participantes ( $n=612,39,5 \%$ ), enquanto a Região Sul foi a que teve menor número ( $\mathrm{n}=65,4,2 \%)$. Embora não tenha sido possível fazer um levantamento preciso dessa variável, pelo menos $90 \%$ da amostra foi composta por estudantes universitários(as), mas houve participantes de outros níveis de escolaridade. A amostra total incluiu $1.048(67,7 \%)$ mulheres, 500 (32,3\%) homens e um(a) participante que não informou o sexo. A amostra possui um terço de estudantes de Psicologia $(\mathrm{n}=553,35,7 \%)$. Contudo, há um elevado número de estudantes de áreas correlatas da Administração e Gestão ( $n=360,23,2 \%$ ). Apesar de mais da metade da amostra se concentrar nessas duas áreas, houve diversidade, pois foram incluídos 85 diferentes cursos superiores, de pós-graduação, profissões.

Os(as) participantes eram basicamente heterossexuais $(n=1.452,93,7 \%)$, porém com um número razoável de bissexuais $(n=27,1,7 \%)$ e homossexuais $(n=23,1,5 \%)$. Três $(0,2 \%)$ participantes afirmaram possuir outra orientação sexual. Sabe-se que, muitas vezes, por se tratar de uma informação obtida por autorrelato, este dado pode não corresponder à realidade, o que também pode ser percebido pelo número de casos omissos $(n=44,2,8 \%)$. A maioria da amostra $(n=1.165,75,2 \%)$ estava solteira quando os dados foram coletados, seguidos por casados(as) $(n=246, \quad 15,9 \%), \quad$ noivos(as) $\quad(n=60, \quad 3,9 \%)$, divorciados(as) $(n=34,2,2 \%)$ e viúvos(as) $(n=4,0,3 \%)$. Trinta e duas $(2,1 \%)$ pessoas encontravam-se em outra situação, enquanto oito $(0,5 \%)$ não responderam à questão. Assim, diferentemente de grande parte dos estudos da área, este teve a participação de pessoas não envolvidas em relacionamentos. De qualquer forma, praticamente metade $\operatorname{dos}($ as $)$ solteiros(as) $(n=596$, $51,2 \%)$ e dos(as) divorciados(as) $\quad(n=16, \quad 47,1 \%)$ afirmaram estar namorando. A maioria dos(as) participantes não possuía filhos(as) $(n=1.242,80,2 \%)$ na ocasião da coleta de dados. Uma melhor descrição dos participantes é feita por Cassepp-Borges (2010).

\section{Instrumentos}

Foi aplicado um questionário com perguntas demográficas, como sexo, data de nascimento, curso [caso fosse universitário(a)] e orientação sexual. Nesse questionário, foi solicitado que $\mathrm{o}$ (a) participante escrevesse o nome de alguma pessoa que amasse. Preferencialmente, o(a) participante deveria indicar alguém com quem compusesse um par amoroso. A partir daí, foram feitas perguntas sobre o tipo e o tempo de relacionamento com essa pessoa.

A RelAS, conforme já mencionado, é composta por 7 itens que medem o construto da satisfação no relacionamento. Trata-se de um instrumento unidimensional (Hendrick, 1988). Foi mantida sua estrutura com 7 pontos na escala tipo Likert, sendo que o ponto 1 representava discordância e $\quad$ o 7 concordância. Por ser uma escala curta, ela foi traduzida diretamente para o português, sem a realização de tradução reversa. A tradução, no entanto, foi revisada por duas pessoas bilíngues. A RelAS foi respondida tendo como base o relacionamento com a pessoa mencionada pelo(a) participante no questionário demográfico. Um exemplo de item é "O quanto seu relacionamento é bom em comparação com os demais?"

\section{Procedimentos}

Apesar de não haver um controle sobre o número exato, as aplicações dos questionários, de maneira geral, foram coletivas, em universidades. Em alguns casos, houve aplicações individuais. Os dados foram coletados entre 08/05/2008 e 05/12/2009. O projeto desta pesquisa foi aprovado pelo Comitê de Ética em Pesquisa, atendendo às recomendações da resolução 196/1996 do Conselho Nacional de Saúde. Todos(as) os(as) participantes foram solicitados(as) a assinar um termo de consentimento livre e esclarecido em duas vias, sendo que uma via permaneceu com o(a) participante e a outra foi entregue ao pesquisador.

Uma tarefa dos(as) participantes foi escolher uma pessoa que amassem, para responder à pesquisa baseando-se no relacionamento com essa pessoa. Pediu-se que, de preferência, fosse escolhido alguém com quem eles compusessem um par amoroso. Por esta razão, a maioria dos(as) participantes baseou-se em um relacionamento romântico estável $(n=831,53,6 \%$ ), nome dado ao agrupamento das categorias namorado(a), noivo(a), casado(a) e morando juntos(as). Provavelmente por serem jovens, a maioria dos(as) participantes em um relacionamento romântico estável $(n=473,56,9 \%)$ estava namorando. Praticamente um quinto $\operatorname{dos}(\mathrm{as})$ respondentes preencheram os instrumentos pensando em um objeto amado que não 
possuíam $(n=301,19,4 \%)$, sendo um amor platônico $(n=118,39,2 \%)$ ou um(a) ex $(n=183,60,8 \%)$. Um número considerável de participantes se baseou em um relacionamento romântico não-estável ("ficantes", e relacionamento sem compromisso, por exemplo; $n=166,10,7 \%$ ), e também houve pessoas que responderam baseando-se em alguém com quem possuíam parentesco $(n=69,4,5 \%)$. O fato de não estar vivenciando uma relação romântica não foi um critério de exclusão da amostra. Qualquer tipo de relacionamento pode ser avaliado com a RelAS, e espera-se que propriedades psicométricas como confiabilidade e estrutura fatorial se mantenham estáveis mesmo para participantes que basearam-se no relacionamento com seus pais, por exemplo.

\section{Análise dos dados}

Inicialmente, foi realizada uma análise fatorial exploratória da RelAS. O método utilizado para determinar o número de fatores foi a Análise dos Componentes Principais, seguindo-se os critérios de Kaiser (o número máximo de fatores extraídos deve possuir autovalores maiores do que um), de Harman (todo fator deve explicar pelo menos 3\% da variância total da escala), da inspeção visual do scree plot (entendendo-se o gráfico dos autovalores como uma montanha e um chão, aquilo que faz parte da montanha é considerado como fator, e aquilo que faz parte do chão não é), da análise paralela (comparandose os autovalores encontrados empiricamente com autovalores aleatórios) e pelo critério teórico previsto para cada instrumento (Pasquali, 2005). A fatorabilidade da matriz foi analisada pelo valor do índice Kaiser-Meyer-Olkin (KMO). Embora relatado, o teste de esfericidade de Bartlett não foi levado em consideração, por ser um indicador muito sensível ao tamanho da amostra. Para essas análises, usou-se o método pairwise para lidar com os casos omissos, pois ele aproveita aquilo que o sujeito respondeu para calcular a matriz de correlações. Escolheu-se aquele item com menor número de respondentes para determinar o tamanho da amostra para efeitos de realização da análise paralela.

Posteriormente foi realizada a extração Principal Axis Factoring $(P A F)$ para determinar as cargas fatoriais dos itens. Utilizou-se a covariância residual não explicada com a finalidade de revisar a decisão sobre o número de fatores. Esse é um critério a posteriori, pois é feito depois da extração dos fatores com a $P A F$. A consistência interna foi calculada por meio dos coeficientes alfa de Cronbach e lambda 2 de Guttman.

A dificuldade (parâmetro b) e a discriminação (parâmetro a) dos itens foram analisadas à luz da Teoria de Resposta ao Item (TRI) (Pasquali, 2007), com o software PARSCALE 4.1 for Windows $($ De acordo com Vendramini e Dias (2005), itens com parâmetro $a$ menor do que 0,30 ou com o parâmetro $b$ fora da faixa na qual se espera que ele se situe (entre 2,95 e +2,95) seriam considerados problemáticos. Baker (2001), no entanto, sugere categorias de discriminação, tratando uma discriminação inferior a 0,64 como baixa e até 1,34 como moderada. Os índices de discriminação altos seriam superiores a 1,35. Os itens ainda deveriam estar distribuídos em diferentes faixas de dificuldade (Pasquali, 2004). Chama-se a atenção que, em análise de instrumentos com escala tipo Likert pela TRI, quando um item é classificado como fácil ou difícil, está-se referindo à facilidade ou à dificuldade que os(as) participantes têm de concordar ou discordar das afirmativas, mais especificamente ao nível de $\theta$ necessário para concordar com o item (Nunes \& colaboradores, 2008). A discriminação se refere à capacidade dos itens para separar participantes com diferentes níveis do traço latente.

\section{Resultados}

Para responder à pesquisa, a maioria dos(as) participantes baseou-se em um relacionamento romântico estável $(n=831,53,6 \%)$, nome dado ao agrupamento das categorias namorado(a), noivo(a), casado(a) e morando juntos(as). Provavelmente por serem jovens, a maioria dos(as) participantes em um relacionamento romântico estável estava namorando $(n=473,56,9 \%)$. Praticamente um quinto dos(as) respondentes preencheram os instrumentos pensando em um objeto amado que não possuíam $(n=301$, $19,4 \%)$, sendo um amor platônico $(n=118,39,2 \%)$ ou um(a) ex $(n=183,60,8 \%)$. Um número considerável de participantes se baseou em um relacionamento romântico não-estável ("ficantes", e relacionamento sem compromisso, por exemplo; $n=166,10,7 \%$ ), e também houve pessoas que responderam baseando-se em alguém com quem possuíam parentesco $(n=69$, $4,5 \%)$.

Com relação às análise psicométricas, primeiramente foi realizada uma análise dos componentes principais da $\operatorname{Rel} A S$, com a finalidade de determinar a fatorabilidade $\mathrm{da}$ matriz de intercorrelações e o número de fatores. Os resultados indicaram um valor de $\mathrm{KMO}$ de 0,883 , com o teste de esfericidade de Bartlett significativo $\left(\chi^{2}=5.202,785\right.$, $g l=21, p<0,001)$, indicando que a matriz de correlações é fatorável. Ademais, procurou-se estabelecer o número de componentes da RelAS por diversos critérios (Tabela 1). Concluiu-se por aceitar o critério teórico de um único fator, que é a solução mais parcimoniosa e coerente com a inspeção visual do scree 
plot (Figura 1) e com a análise paralela (Tabela 2).

Chama-se a atenção para o fato de que o critério de
Kaiser é fraco para instrumentos com poucos itens como a RelAS.

Tabela 1. Critérios considerados na decisão do número de fatores a extrair da RelAS

\begin{tabular}{|c|c|c|c|c|c|}
\hline Critério & $\begin{array}{l}\text { Número de } \\
\text { fatores }\end{array}$ & \multicolumn{4}{|c|}{ Comentários } \\
\hline Kaiser (K-1) & $\begin{array}{l}\text { Pelo menos } \\
\quad 2\end{array}$ & \multicolumn{4}{|c|}{$\begin{array}{l}\text { O segundo autovalor é bastante próximo de um, o que sugere que o } \\
\text { segundo fator tem pouca força (Tabela 2). }\end{array}$} \\
\hline Harman & 6 & \multicolumn{4}{|c|}{ Critério fraco, devido à baixa quantidade de itens (Tabela 2). } \\
\hline $\begin{array}{l}\text { Inspeção visual do } \\
\text { Scree plot }\end{array}$ & 1 & \multicolumn{4}{|c|}{ A partir do segundo autovalor, o desenho vira uma reta (Figura 1). } \\
\hline Análise paralela & 1 & \multicolumn{4}{|c|}{$\begin{array}{l}\text { O segundo autovalor empírico é inferior ao segundo autovalor aleatório } \\
\text { (Figura } 1 \text { e Tabela 2). }\end{array}$} \\
\hline Critério teórico & 1 & \multicolumn{4}{|c|}{ Satisfação no relacionamento } \\
\hline Componente & & $\begin{array}{c}\text { Autovalores } \\
\text { empíricos }\end{array}$ & $\begin{array}{c}\text { Autovalores } \\
\text { aleatórios }\end{array}$ & $\begin{array}{c}\% \text { de } \\
\text { variância }\end{array}$ & $\%$ acumulado \\
\hline 1 & & 3,87 & 1,10 & 55,3 & 55,3 \\
\hline 2 & & 1,03 & 1,06 & 14,7 & 70,0 \\
\hline 3 & & 0,73 & 1,03 & 10,5 & 80,5 \\
\hline 4 & & 0,56 & 1,00 & 8,0 & 88,5 \\
\hline 5 & & 0,30 & 0,97 & 4,3 & 92,9 \\
\hline 6 & & 0,30 & 0,94 & 4,3 & 97,1 \\
\hline 7 & & 0,20 & 0,91 & 2,9 & 100,0 \\
\hline
\end{tabular}

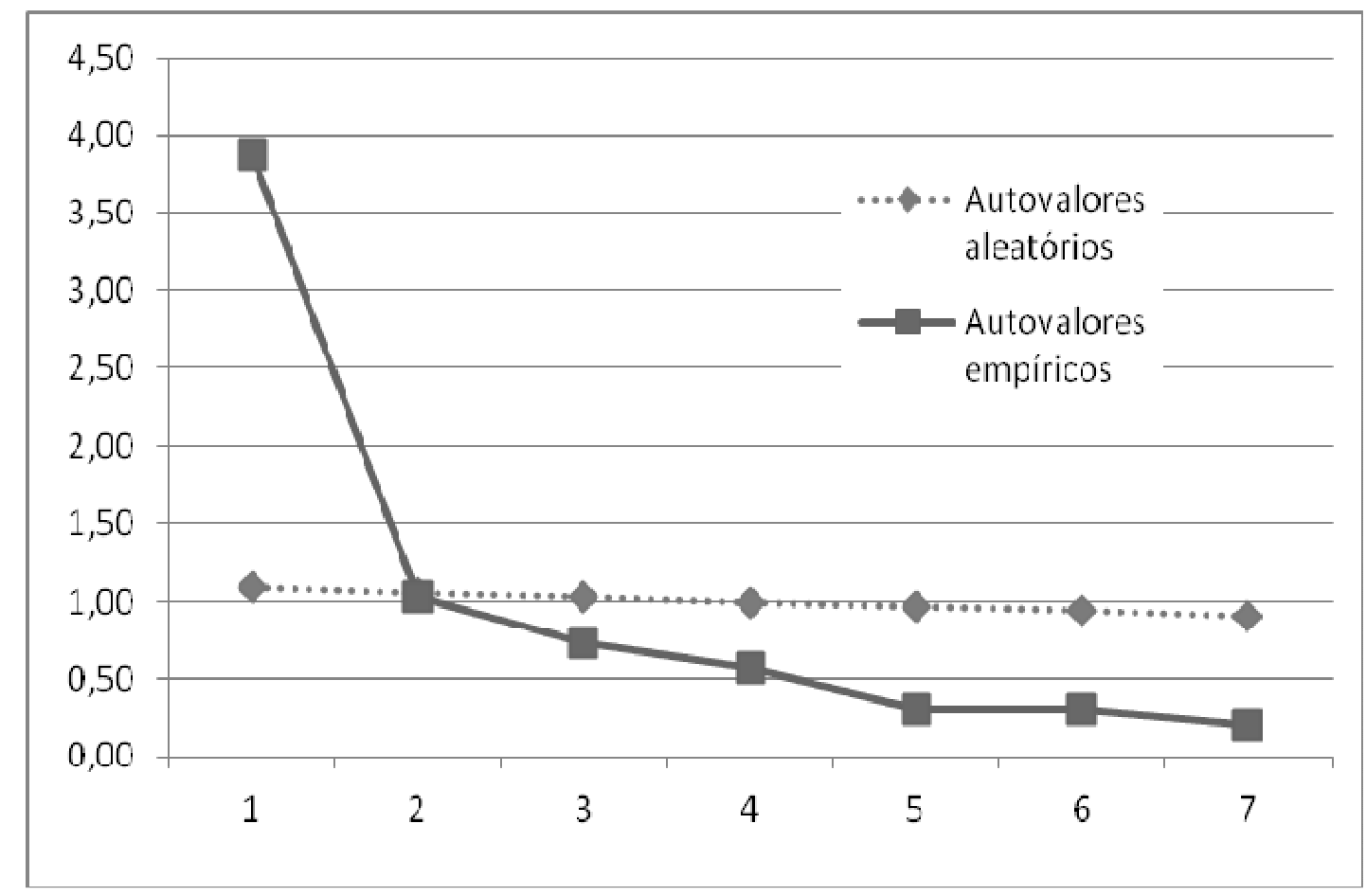

Figura 1. Scree plot dos autovalores empíricos e aleatórios da RelAS 
Depois de decidir por um fator único, consoante com a análise paralela, com a inspeção visual do scree plot e com a teoria, procedeu-se à extração dele. Chama-se a atenção para o fato de que não existe rotação quando se realiza a análise de um fator único. Foi utilizado o método de extração $P A F$, que permite realizar a inferência de que o traço latente é o causador do comportamento descrito pelos itens. De acordo com a Tabela 3, pode-se notar que as cargas fatoriais mais baixas referem-se aos dois itens que devem ser invertidos na escala (RelAS_4 e RelAS_7). Apesar disso, quatro itens possuem cargas fatoriais superiores a 0,80 , o que pode ser considerado excelente. Mesmo com poucos itens, a escala mostrou um bom nível de consistência interna $(\alpha=0,85)$.

Tabela 3. Coeficientes pattern e comunalidades da RelAS com o método Principal Axis Factoring

\begin{tabular}{ccc}
\hline Item & Carga & $h^{2}$ \\
\hline RelAS_2 & 0,91 & 0,82 \\
RelAS_3 & 0,85 & 0,72 \\
RelAS_5 & 0,85 & 0,71 \\
RelAS_1 & 0,81 & 0,66 \\
RelAS_6 & 0,53 & 0,28 \\
RelAS_4 & $-0,44$ & 0,20 \\
RelAS_7 & $-0,34$ & 0,12 \\
Carga média & 0,68 & \\
Alfa de Cronbach & 0,847 & \\
Lambda 2 de Guttman & 0,860 & \\
Itens & 7 & \\
\hline
\end{tabular}

Nota: Variância explicada: 1 fator $=50,05 \% ; 2$ fatores $=58,63 \%$.

Covariância residual: 1 fator $=14 \% ; 2$ fatores $=0 \%$.

$\mathrm{O}$ valor das comunalidades $\left(b^{2}\right)$ pode diferir do quadrado da carga fatorial por motivo de arredondamento.

A estrutura fatorial com um fator único pode ser corroborada pelo nível de apenas $14 \%$ de covariância residual não explicada. Não valeria a pena inserir outro fator para explicar somente $3(14 \%)$ covariâncias residuais, pois isso dobraria a complexidade da solução fatorial, ferindo o corolário da parcimônia. A melhora dos níveis de variância explicada também não seria tão relevante caso se optasse pela solução com 2 fatores. $\mathrm{O}$ ganho em variância explicada também é pequeno quando acrescenta-se um segundo fator.

A análise de TRI da RelAS foi feita utilizando-se o tratamento listwise para casos omissos, restando 1.462 participantes que responderam aos sete itens da escala. Contudo, a matriz das intercorrelações se mostrou singular, ou seja, seu determinante foi igual a zero, impossibilitando o cálculo da inversa da matriz. Dessa maneira, a solução encontrada para resolver essa situação foi alterar o parâmetro "Scale" (constante D) para a realização das análises, de 1,7 para 1,0. A constante $D$ tem seu valor fixado em 1,7 para que sua métrica logística se equipare à da métrica normal. Uma vez que os dados não seguem essa métrica, o modelo logístico fez-se necessário. Ao fixar a constante D em 1,0, elimina-se a correção para a métrica normal para utilizar-se o modelo logístico (Pasquali, 2007).

Os resultados (vide Tabela 4) indicaram que todos os itens são discriminativos, pois o valor mais baixo no parâmetro a foi 1,09 e a média foi de 2,55. Assim, entende-se que os itens são capazes de separar participantes satisfeitos(as) de insatisfeitos(as) com o relacionamento. Os itens ainda são relativamente "fáceis". Apenas o item RelAS_7 apresentou uma dificuldade (parâmetro b) próxima de zero. Mesmo sendo o item com carga fatorial mais baixa, sua presença no instrumento se justifica pelo fato de ser o item mais difícil, que avalia uma parcela da amostra com maior satisfação no relacionamento. Em média, a dificuldade dos itens foi de $-0,86$. Pode-se dizer que a escala é composta por itens médios e fáceis, ou seja, não é necessário um grau muito elevado de satisfação no relacionamento para concordar com as afirmativas. 
Tabela 4. Discriminação e dificuldade dos itens da RelAS

\begin{tabular}{lcccc}
\hline Item & Discriminação & Erro padrão & Dificuldade & Erro padrão \\
\hline RelAS_1 & 3,445 & 0,103 & $-0,828$ & 0,037 \\
RelAS_2 & 3,768 & 0,124 & $-0,686$ & 0,033 \\
RelAS_3 & 2,906 & 0,094 & $-0,988$ & 0,036 \\
RelAS_4 & 1,094 & 0,037 & $-1,216$ & 0,055 \\
RelAS_5 & 3,224 & 0,093 & $-0,699$ & 0,034 \\
RelAS_6 & 1,815 & 0,054 & $-1,512$ & 0,047 \\
RelAS_7 & 1,644 & 0,039 & $-0,058$ & 0,053 \\
Média & 2,556 & & $-0,855$ & \\
\hline
\end{tabular}

A Figura 2 apresenta a curva de informação da RelAS. A escala é informativa para aqueles(as) participantes $\operatorname{com} \theta$ abaixo de 0,85 , sugerindo que a informação é baixa para participantes com elevada satisfação no relacionamento. De qualquer forma, a informação da escala é maior do que o erro de medida para participantes com escore $\theta$ aproximadamente entre -3 e $+0,9$. Assim, a escala é útil para os sujeitos localizados entre o percentil 0 e o percentil 80. Isso significa que a RelAS tem dificuldade de discriminar a quantidade de satisfação no relacionamento dos sujeitos mais satisfeitos. $\mathrm{O}$ fato de ser composta em sua maioria por itens fáceis alterou a informação da escala. Mas, como indica a Figura 3, mesmo que a RelAS tenha falhado no teste de normalidade (KolmogorovSmirnov $=3,64, \mathrm{p}<0,001)$, a TRI demonstra que a distribuição dos escores foi praticamente normal em torno da média.

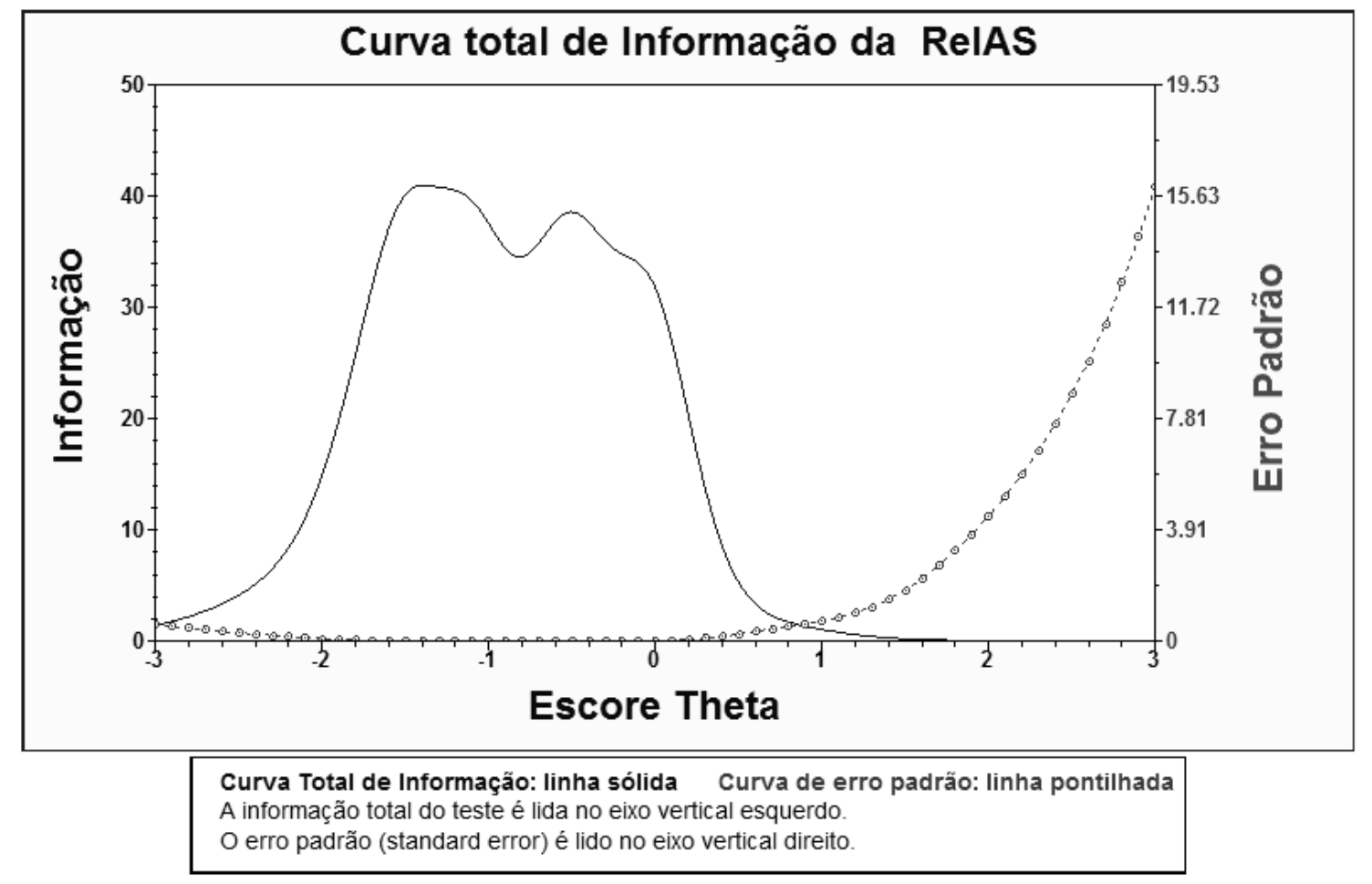

Figura 2. Curva de informação da RelAS

\section{Discussão}

O tema da satisfação no relacionamento é atual, relevante e complexo. Conhecer as variáveis que afetam a satisfação no relacionamento em diferentes contextos é algo de extrema importância para a ciência psicológica e para a aplicação prática da mesma. Contudo, para que esse passo seja dado, é crucial que se tenha instrumentos de medida calibrados e válidos. O estudo da satisfação no relacionamento depende de testes construídos com rigor científico (ScorsoliniComin \& Santos, 2010b).

A RelAS é uma escala útil por ser breve, precisa e informativa. $O$ fato de ter apenas sete itens facilita sua aplicação e correção. Os níveis de precisão se mantiveram, pois o valor de alfa de 0,85 , é muito 
parecido com o do estudo original $(\alpha=0,86$, Hendrick, 1988). Este estudo tem a limitação de não ter verificado a confiabilidade teste-resteste da RelAS, o que poderia trazer mais uma evidência da precisão. É um instrumento que, embora menor do que a $D A S$ (Spanier, 1976), avalia o mesmo construto. A $D A S$ possui quatro dimensões (consenso, satisfação, coesão e expressão afetiva) e é o instrumento mais utilizado para avaliar satisfação no relacionamento. Suas dimensões, no entanto, são bastante correlacionadas. A Escala Fatorial de Satisfação em Relacionamento também possui seus dois fatores correlacionados em 0,36 (Wachelke e colaboradores, 2004). Assim, é plausível inferir um fator geral de satisfação no relacionamento. Apesar de não ser possível fazer essa afirmação sustentada nos dados empíricos desta pesquisa, uma comparação dos itens da RelAS com as dimensões de ambas escalas sugere que a RelAS provavelmente esteja medindo esse fator geral. Faz-se necessário verificar a correlação com uma escala que avalie o mesmo construto para melhor aferir a validade convergente da RelAS.

\section{Ajuste Gaussiano para os Escores de habilidade da ReIAS}

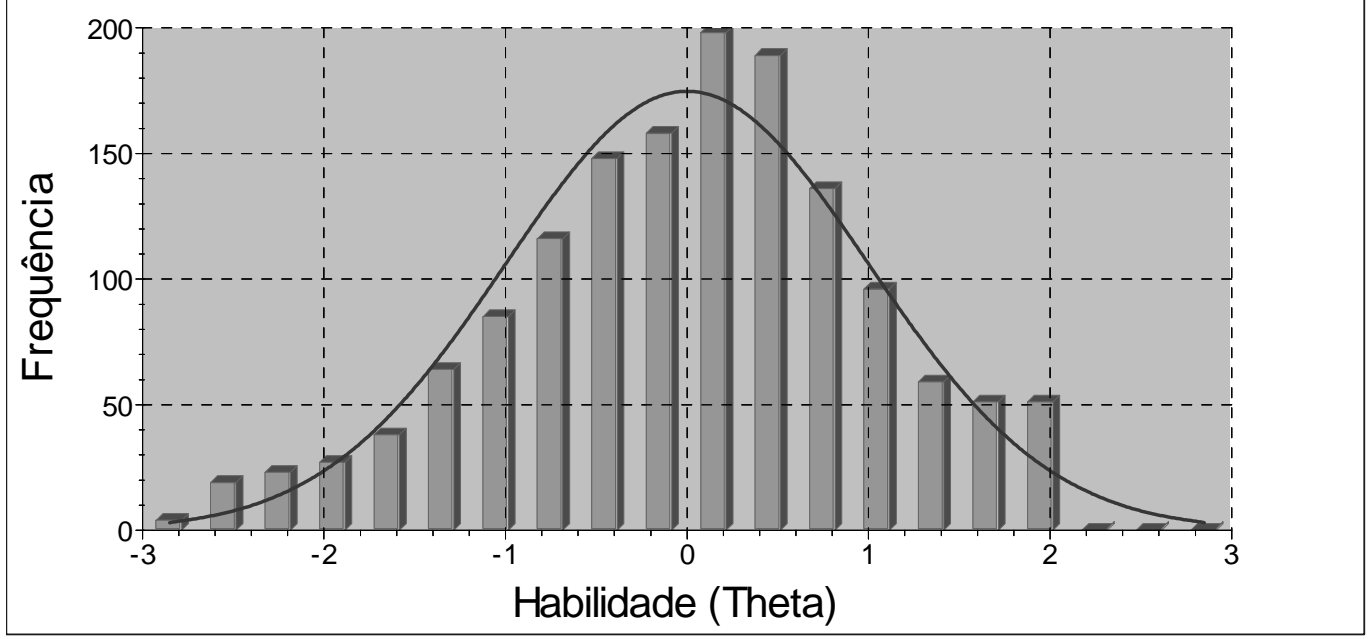

Histograma das habilidades

Os escores de habilidade foram transformados para ter média $=0$ e desvio padrâ

A área abaixo da curva normal é igual à área total do histograma.

Figura 3. Distribuição dos escores da RelAS em torno da média

Contudo, pelo fato da RelAS ser informativa para sujeitos aproximadamente entre o percentil zero e 80, torna-se um teste útil para uso clínico, pois avalia a faixa com problemas de satisfação no relacionamento, na qual é favorável realizar intervenções. Seria necessária a criação de alguns itens com maior nível de dificuldade, para avaliar o espectro mais satisfeito da população. A RelAS, no entanto, possui itens discriminativos e ainda mostrou-se válida e fidedigna como instrumento de medida.

A medida da satisfação pode permitir intervenções psicológicas com maior sustentação científica. Um elevado grau de insatisfação com o relacionamento pode estar associado a um baixo bem-estar ou mesmo com amor patológico. A RelAS é uma escala para ser usada diversas vezes em um tratamento psicoterápico, para acompanhar a evolução da satisfação no relacionamento e promover a melhoria na qualidade de vida. Agências de relacionamento que desejem oferecer aos seus clientes um trabalho sério podem medir a satisfação nos relacionamentos arranjados pela agência, de maneira a avaliar que tipo de casal tem mais chance de sucesso.

Conforme já demonstrado em outros estudos estrangeiros (Hendrick, 1988; Hendrick \& colaboradores, 1998), a RelAS possui adequados indicadores psicométricos. É um instrumento capaz de medir de maneira breve e precisa a satisfação no relacionamento. A estrutura unifatorial também é uma propriedade interessante, pois a parcimônia é um corolário importante da análise fatorial. No Brasil, a RelAS replicou as boas propriedades conhecidas no exterior, e espera-se que em breve esteja disponível para uso prático.

\section{Referências}

Antoine, P., Christophe, V. \& Nandrino, J. L. (2008). Dyadic Adjustment Scale: clinical interest of a revision and validation of an abbreviated form. 
Encephale - Revue de Psychiatrie Clinique Biologique et Therapentique, 34(1), 38-46.

Baker, F. B. (2001). The basics of item response theory. Washington, DC: ERIC.

Cassepp-Borges, V. (2010). Amor e construtos relacionados: evidências de validade de instrumentos de medida no Brasil. (Tese de Doutorado). Brasília, Distrito Federal: Universidade de Brasília.

Cassepp-Borges, V. \& Teodoro, M. L. M. (2009). Versión reducida de la Escala Triangular del Amor: características de sentimiento en Brasil. Revista Interamericana de Psicología, 43(1), 30-38.

Conselho Federal de Psicologia (2011). Sistema de avaliação de testes psicológicos - Lista de testes com parecer favorável. Extraído do sítio http://www2.pol.org.br/satepsi/sistema/admin.cf m no dia 11/01/2011.

Conselho Nacional de Saúde (1996). Resolução 196, de 10 de outubro de 1996. Brasília: Diário Oficial da União.

Cramer, D. (2006). How a supportive partner may increase the relationship satisfaction. British Journal of Guidance \& Counseling, 34(1), 117-131.

De Andrade, A. L. \& Wachelke, J. F. R. (2006). Pesquisa psicológica via internet: satisfação em relacionamentos de casal e bem-estar psicológico, validação e parâmetros psicométricos. Anais da $58^{a}$ Reunião Anual da SBPC. Florianópolis.

Dela Coleta, M. F. (1989). A medida da satisfação conjugal: Adaptação de uma escala. Psico, 18(2), 90-112.

Dinkel, A. \& Balck, F. (2005). An evaluation of the German Relationship Assessment Scale. Swiss Journal of Psychology, 64(4), 259-263.

Dinkel, A. \& Balck, F. (2006). Psychometric analysis of the German Dyadic Adjustment Scale. Zeitschrift fur Psychologie, 214(1), 1-9.

Funk, J. L. \& Rogge, R. D. (2007). Testing the ruler with Item Response Theory: increasing precision of measurement for relationship satisfaction with the couples satisfaction índex. Journal of Family Psychology, 21(4), 572-583.

Gable, S. L. \& Poore, J. (2008). Which thoughts count? Algorithms for evaluating satisfaction in relationships. Psychological Science, 19(10), 10301036.
Hendrick, S. S. (1988). A generic measure of relationship satisfaction. Journal of Marriage and the Family, 50, 93-98.

Hendrick, S. S., Dicke, A. \& Hendrick, C. (1998). The Relationship Assessment Scale. Journal of Social and Personal Relationships, 15(1), 137-142.

Hernandez, J. A. E. (2008). Avaliação estrutural da Escala de Ajustamento Diádico. Psicologia em Estudo, 13(3), 593-601.

Hundertmark, J., Esterman, A. Ben-Tovim, D., Austin M. A. \& Dougherty, M. (2007). The South Australian couples sildenafil study: double-blind, parallel-group randomized controlled study to examine the psychological and relationship consequences of sildenafil use in couples. Journal of Sexual Medicine, 4(4), 1126-1135.

Instituto Brasileiro de Geografia e Estatística (2011). Divisão regional. Extraído do sítio http://www.ibge.gov.br/home/geociencias/geogr afia/default_div_int.shtm?c=1 no dia 11/01/2011.

Lemieux, R. \& Hale, J. L. (2000). Intimacy, passion and commitment among married individuals: further testing of the Triangular Theory of Love. Psychological Reports, 87, 941-948.

Lesch, E. \& Engelbrecht, S. K. (2008). The reliability of the Dyadic Satisfaction (DS) subscale in a lowincome semi-rural South African community. Journal of Psychology in Africa, 18(2), 245-248.

Masuda, M. (2003). Meta-analyses of love scales: do various love scales measure the same psychological constructs? Japanese Psychological Research, 45(1), 2537.

Norgren, M. B. P., Souza, R. M., Kaslow, F., Hammerschmidt, H. \& Sharlin, S. A. (2004). Satisfação conjugal em casamentos de longa duração: uma construção possível. Estudos de Psicologia (Natal), 9(3), 575-584.

Nunes, C. H. S. S., Primi, R., Nunes, M. F. O., Muniz, M., Cunha, T. F. \& Couto, G. (2008). Teoria de Resposta ao Item para otimização de escalas tipo likert - um exemplo de aplicação. Revista Iberoamericana de Evaluación Psicologica, 25(1), 51-79.

Pasquali, L. (2004). Psicometria: teoria dos testes na psicologia e na educação. Petrópolis: Vozes.

Pasquali, L. (2005). Análise fatorial para pesquisadores. Brasília: LabPAM.

Pasquali, L. (2007). Teoria de Resposta ao Item: teoria, procedimentos e aplicações. Brasília: LabPAM. 
Perlin, G. dal B. (2001). Casais que trabalham e são felizes: mito on realidade? (Dissertação de Mestrado). Brasília, Distrito Federal: Universidade de Brasília.

Perlin, G. dal B. (2006). Casamentos contemporâneos: um estudo sobre os impactos da interação família-trabalho na satisfação conjugal. (Tese de Doutorado). Brasília, Distrito Federal: Universidade de Brasília.

Perlin, G. dal B. \& Diniz, G. (2005). Casais que trabalham e são felizes: mito ou realidade? Psicologia Clínica, 17(2), 15-29.

Scorsolini-Comin, F. \& Santos, M. A. (2010a). Satisfação com a vida e satisfação diádica: correlações entre construtos de bem-estar. PsicoUSF, 15(2), 249-256.

Scorsolini-Comin, F. \& Santos, M. A. (2010b). Satisfação conjugal: revisão integrativa da literatura científica nacional. Psicologia: Teoria e Pesquisa, 26(3), $525-531$

Shek, D. T. L. \& Cheung, C. K. (2008). Dimensionality of the Chinese Dyadic Adjustment Scale based on confirmatory factor analyses. Social Indicators Research, 86(2), 201-212.
Spanier, G. B. (1976). Measuring dyadic adjustment: new scales for assessing the quality of marriage and similar dyads. Joumal of Marriage and the Family, 38(1), 15-28.

Vendramini, C. M. M. \& Dias, A. S. (2005). Teoria de Resposta ao Item na análise de uma prova de estatística em universitários. Psico-USF, 10(2), 201 210.

Wachelke, J. F. R., De Andrade, A. L., Cruz, R. M., Faggiani, R. B. \& Natividade, J. C. (2004). Medida da satisfação do relacionamento de casal. PsicoUSF, 9(1), 11-18.

Wachelke, J. F. R., De Andrade, A. L., Souza, A. M. \& Cruz, R. M. (2007). Estudo complementar da validade fatorial da Escala Fatorial de Satisfação em Relacionamento e predição de satisfação global com a relação. Psico-USF, 12(2), 221-225.

Recebido em 28/03/2011

Reformulado em 10/07/2011

Aprovado em 06/09/2011

Nota: Embora conhecida como RAS no exterior, preferiu-se adotar a sigla RelAS, para diferenciar da Escala de Assertividade Rathus, que possui a sigla $\mathrm{R} A S$.

Trabalho oriundo da tese de doutorado do primeiro autor, orientado pelo segundo, no programa de pós-graduação em Psicologia Social, do Trabalho e das Organizações da Universidade de Brasília.

Auxílio: CAPES.

Sobre os autores:

Vicente Cassepp-Borges é graduado em Psicologia pela Universidade do Vale do Rio dos Sinos (2006) e doutor em Psicologia Social, do Trabalho e das Organizações (PSTO) pela Universidade de Brasília (UnB). Recebeu o Prêmio Estudantil da Sociedade Interamericana de Psicologia em 2007. Atualmente, é professor adjunto da Universidade Federal da Grande Dourados (UFGD).

Luiz Pasquali possui Graduação em Filosofia pelo Instituto Franciscano de Filosofia, Graduação em Pedagogia pela Universidade Católica de Petrópolis, e Graduação, Mestrado e Doutorado em Psychologie et des Sciences de l'Éducat pela Université Catholique de Louvain. Atualmente, é professor pesquisador associado da Universidade de Brasília (UnB). Tem experiência na área de Fundamentos e Medidas da Psicologia. 